KFKI-1980-18

L.P. HUGHSTON

\title{
THE TWISTOR PARTICLE PROGRAMME
}

\section{Hungarian Academy of Sciences}

CENTRAL

RESEARCH

INSTITUTE FOR

PHYSICS

\section{BUDAPEST}


KFKI-1980-18

\section{THE TWISTOR PARTICLE PROGRAMME}

L.P. Hughston

The Mathematical Institute, University of Oxford, oxford, England

Submitted to Journal of High-Energy Physics

HU ISSN O368 5330

ISBN $9633 \quad 371 \quad 646 \quad 2$ 


\section{ABSTRACT}

Classification schemes for elementary particles are discussed using methods of twistor theory, with particular emphasis on the relationship of twistor theory to the Weinberg-Salam model and the Georgi-Glashow SU(5) model.

\section{АННОТАЦИЯ}

Изучапись системы классификации элементарных частиц с помощью методов теории твисторов, подчеркивая главным образом взаимосвязь между теорией твисторов и моделями Вейнберга - Салама и SU/5/ Джорджи-Глашоу.

\section{KIVONAT}

Tvisztorelméleti módszerek segitségével részecskeosztályozási rendszereket tanulmányozunk, fơképpen a tvisztorelméletnek és a Weinberg-Salam, valamint a Georgi-Glashow-féle SU(5) modellnek a kapcsolatát kiemelve. 


\section{THE $n$-TWISTOR INTERNAL SYMMETRY GROUPS}

The notion of internal symmetries plays an important role in particle physics, and one of the objectives of the twistor particle programme is to gain some understanding of the origins of these internal symmetries. A convenient place to begin is with the analysis of classical massive systems with spin. Such a system is specified by its relativistic momentum $\mathrm{P}^{\mathrm{a}}$ and its angular momentum $\mathrm{M}^{\mathrm{ab}}$ given with respect to some choice of origin. Any classical massive system can be regarded as being composed of two or more twistors. If these twistors are labelled $z_{i}^{\alpha}$ with $i=1 \ldots n$, then the momentum and angular momentum of the system are represented by a kinematic twistor $A^{\alpha \beta}$ determined as follows:

$$
A^{\alpha \beta}=2 z_{i}^{(\alpha} I^{\beta) \gamma} \bar{z}_{\gamma^{\prime}}^{i}
$$

where $I^{\alpha \beta}$ is the infinity twistor. The kinematic twistor describes the momentum and angular momentum of the system according to the scheme,

$$
A^{\alpha \beta}=\left(\begin{array}{cc}
-2 i \mu^{A B} & P_{B^{\prime}}^{A} \\
P_{A^{\prime}}{ }^{B} & 0
\end{array}\right) \text {, }
$$

where $\mathrm{P}^{A A^{\prime}}$ is the momentum, and $\mu^{\mathrm{AB}}$ is the angular momentum spinor, which determines the angular momentum by means of the relation

$$
M^{a b}=\mu^{A B} \varepsilon^{A^{\prime} B^{\prime}}+\bar{\mu}^{-A^{\prime} B^{\prime}} \varepsilon^{A B} .
$$

For any given value of $A^{\alpha \beta}$ it is possible to choose $z_{i}^{\alpha}$ in many different ways such that equation (1) holds. The n-twistor internal symmetry group is defined to be the set of all linear transformations of $z_{i}^{\alpha}$ and $\bar{z}_{\alpha}^{i}$ that satisfy these two conditions:
a) $A^{\alpha \beta}$ is invariant
b) the conjugacy relations between $z_{i}^{\alpha}$ and $\bar{z}_{\alpha}^{i}$ are preserved. 
Such transformations turn out to be of the form

$$
\left\{\begin{array}{l}
z_{i}^{\alpha} \longrightarrow U_{i}^{j}\left(z_{j}^{\alpha}+I^{\alpha \beta} \Lambda_{j k} \bar{z}_{\beta}^{k}\right) \\
\bar{z}_{\alpha}^{i} \longrightarrow \bar{U}_{j}^{i}\left(\bar{z}_{\alpha}^{j}+I_{\alpha \beta} \bar{\Lambda}^{j k_{z} z_{k}^{\beta}}\right),
\end{array}\right.
$$

with $U_{i}^{j}$ unitary and $\Lambda_{j k}$ skew-symmetric. These groups are intimately related to the symmetry groups that arise in elementary particle physics, and a good deal of effort has been put into the analysis of their structure. The expression (1) above appeared originally in Penrose and MacCallum ${ }^{1}$, and there was much discussion of the n-twistor groups shortly thereafter, especially in Penrose's seminars at Birkbeck College, London in 1973. The first systematic investigation of these groups to be published was due to Perjés ${ }^{2}$, who examined many aspects of the 2-twistor and 3-twistor groups. The n-twistor internal symmetry groups have subsequently been the subject of many further investigations by a number of authors, including Penrose $3,4,5, \operatorname{Hodges}^{6}, \operatorname{Tod}^{7,8}, \operatorname{Perjés}^{9}$, Popovich $^{10}$, Perjés and Sparling ${ }^{11}$, Penrose and sparling ${ }^{12}$, Sheppard ${ }^{13}$, Hughston $14,15,16$, Perjés ${ }^{17}$, and others.

\section{TWISTOR QUANTIZATION}

Quantum mechanical observables are introduced by applying the rules of twistor quantization 18 according to which the complex conjugate twistor variables $\overline{\mathrm{z}}_{\alpha}^{i}$ are replaced by the differential operators $\hat{\mathrm{z}}_{\alpha}^{i}$ defined by

$$
\hat{z}_{\alpha}^{i}=-\partial / \partial z_{i}^{\alpha} \text {. }
$$

According to this procedure one obtains the following expression for the operators corresponding to momentum and angular momentum:

$$
\hat{A}^{\alpha \beta}=2 z_{i}^{(\alpha} I^{\beta) \gamma} \hat{Z}_{\gamma^{\prime}}^{i}
$$

where we use a 'hat' on $\hat{A}^{\alpha \beta}$ in order to distinguish it from the corresponding classical quantity appearing in expression (1). More generally we hypothesize that for all physical observables it suffices to consider holomorphic linear differential operators 
with polynomial coefficients. In order to qualify as proper observables such operators must satisfy a Hermitian condition, and must have zero net homogeneity (i.e. must commute with the total Euler operator $\left.-z_{i}^{\alpha} \hat{z}_{\alpha}^{i}\right)$. By an internal observable we mean any observable which commutes with all components of the momentum and the angular momentum. The eigenvalues of internal observables are to be understood as internal quantum numbers; that is to say, we are supposing that the various internal quantum numbers of particle physics (e.g. electric charge, isospin, baryon number, etc.) all arise in this way. One might suspect that there is some connection between the algebra of internal observables and the ntwistor internal symmetry groups, and indeed there is. It is of relevance for this purpose to examine the infinitesimal generators of n-twistor internal symmetries, and a theorem can be proven to the effect that all internal observables can be built up in terms of polynomial expressions in these generators*. A number of illustrative examples of the construction of internal observables will appear in what follows.

3. CONTOUR INTEGRAL FORMULAE

The description of zero rest mass particle states using holomorphic functions of a single twistor is well known. An analogous description for massive particles is also available, first appearing in reference 1, whereby massive particle states are represented by holomorphic functions of two or more twistors. As in the zero rest mass case a contour integral formula can be used in order to extract the relevant space-time field information from the holomorphic function $f\left(z_{j}^{\alpha}\right)$. Let us put

$$
z_{j}^{\alpha}=\left(\omega_{j}^{A}, \pi_{A^{\prime} j}\right)
$$

for the spinor parts of $\mathrm{z}_{j}^{\alpha}$, and write

$$
\hat{\pi}^{A j}=-\partial / \partial \omega_{A j} \cdot
$$

\footnotetext{
${ }^{\star}$ Gratitude is expressed to A.S. Popovich for discussion on this point.
} 
We shall use the convenient notation

$$
\rho_{x} f\left(z_{j}^{\alpha}\right)=f\left(i x^{A A^{\prime}} \pi_{A^{\prime} j}, \pi_{A^{\prime} j}\right)
$$

for the restriction of a function $f\left(z_{j}^{\alpha}\right)$ to the space-time point $x^{A A^{\prime}}$. If the twistor function $f\left(z_{j}^{\alpha}\right)$ happens to correspond to a particle state with a definite set of quantum numbers (i.e. in a simultaneous eigenstate of a suitably complete set of commuting observables constructed as described earlier) then the field associated with that state corresponds to some component of a field multiplet $\phi:::(x)$ given by the contour integral formula

$$
\phi:::(x)=\oint \rho_{x} \hat{\pi} \cdots \pi \cdots f(z) \Delta \pi .
$$

Here $\Delta \pi$ is the wedge product of the $d^{2} \pi$ forms for all the various $\pi_{j}^{A^{\prime}}$ spinors. Note that $\rho_{x}$ is applied only after $\hat{\pi}$ has operated as many times as necessary on $f(z)$. The field $\phi:::(x)$ has both group indices as well as spinor indices; this is why it is referred to as a "multiplet". As indicated above, if $\mathrm{f}\left(\mathrm{z}_{j}^{\alpha}\right)$ is in an eigenstate of a suitably maximal set of compatible observables, the multiplet $\phi:::(\mathrm{x})$ will only admit a single linearly independent component, and this is the space-time field associated to $f\left(z_{j}^{\alpha}\right)$. Thus the twistor function "affords a complete description of a particle, both as regards its external and internal parameters", as Penrose puts it in reference 5. It should be understood, of course, that the description envisaged here is at the first-quantized (i.e. single particle) level, prior in any sense to the switching on of interactions. For further discussion of twistor contour integral formulae see reference 4, reference 15 chapter 5, and $§ 4.1$ in reference 16 .

\section{LEPTONS AS 2-TWISTOR STATES}

Since leptons can be regarded as the simplest of the observed elementary particles, and since two is the minimum number of twistors needed in order to build up a massive state, it is natural to try to build up tentatively a model of leptons based on pairs of twistors. This view is reinforced to some extent when 
one considers that the group $\mathrm{U}(2)$, which plays such an important role in theories of the weak interaction, is a subgroup of the 2-twistor internal symmetry group. Indeed 2-twistor states transform naturally under $\mathrm{U}(2)$, and so one can look to see what sorts of multiplets arise, and whether there is any correlation with the observed families of multiplets. The resulting picture is very encouraging, in certain respects, but not entirely satisfactory. According to the 2-twistor lepton model (I shall describe here one of several distinct models that can be built along these lines) we are to regard the electron and the electron neutrino as comprising an $\mathrm{SU}(2)$ doublet $\phi_{\mathrm{A}}^{i}(\mathrm{x})$. The appropriate contour integral formula* is:

$$
\phi_{A}^{i}(x)=\oint \rho_{x} \hat{\pi}_{A}^{i} f(z) \Delta \pi
$$

The positron and the electron antineutrino form the corresponding antiparticle doublet $\phi_{i}^{A^{\prime}}(\mathrm{x})$, for which the relevant formula is

$$
\phi_{i}^{A^{\prime}}(\mathrm{x})=\oint \rho_{x} \pi_{i}^{A^{\prime}} \mathrm{f}(\mathrm{z}) \Delta \pi \text {. }
$$

It is relatively straightforward to build operators for the following observables: mass, spin, electric charge, lepton number, and 'leptonic isospin'; explicit expressions can be found in rererence $15, \S 8.3$.

As it stands the picture developed thus far has some obvious deficiencies: for example, muons are not included. Muons cannot be realized very naturally within the context of a 2-twistor framework. There are difficulties of a more subtle nature, as well. Perhaps most distressing is the fact that the model is quite incompatible with the Weinberg-Salam mode $2^{19-21}$ in its scheme of assignments of quantum numbers to the leptons. In the Weinberg-Salam model, the left and right hand parts of the electron wave function are assigned to distinct leptonic isospin multiplets: the left hand part belongs to a doublet, along with the neutrino; the right hand part, on the other hand, is taken

\footnotetext{
*By "appropriate contour integral formula" we mean that $f(Z)$ is put in an eigenstate of suitable observables such that if any other combination of $\pi$ 's and $\pi$ 's were used in the formula, the result would necessarily vanish.
} 
to be a singlet state under leptonic isospin. This is impossible in a 2-twistor scheme: a curious degeneracy arises in the 2-twistor case (as was pointed out by K.P. Tod in 1974) leading to the fact that the eigenvalues for spin and leptonic isospin always coincide*. Another difficulty with the 2-twistor scheme is that it allows for only a single neutral vector boson. In order to be consistent with the Weinberg-Salam picture (and, indeed, with neutral current phenomenology) we should prefer two such states - one corresponding to the photon, and another corresponding to the hypothetical neutral z-boson. Again, it is the equivalence between 2-twistor spin and leptonic isospin that gives rise to this difficulty.

\section{A 3-TWISTOR WEINBERG-SALAM SCHEME}

It turns out that the various problems mentioned above can be resolved, provisionally, with the introduction of a somewhat more elaborate scheme based on functions of three twistors - a leptonic isodoublet $\mathrm{z}_{i}^{\alpha}$ and a leptonic isosinglet twistor $\mathrm{Y}^{\alpha}$. Within this scheme it is possible to set up all the states necessary for an account of the Weinberg-Salam model. Formula (11) now refers to the left hand part of the electron and the electron neutrino, the only difference being that $f$ is now taken to be a function of $\mathrm{Y}^{\alpha}$ and $\mathrm{z}_{i}^{\alpha}$. Similarly, formula (12) now refers to the right hand part of the positron and the electron antineutrino, again with $f$ a function of $Y^{\alpha}$ and $z_{i}^{\alpha}$. The right hand part of the electron is given by

$$
\phi_{A^{\prime}}^{i j}=\oint \rho_{x} \hat{\pi}_{A}^{i} \hat{\pi}^{j A} \eta_{A}, f(y, z) \Delta \pi
$$

where $Y^{\alpha}=\left(n^{A}, n_{A},\right)$. The field $\phi_{A}^{i j}$ is an $S U(2)$ singlet since it is skew over the indices $i$ and $j$. Similarly, the left hand part of the positron has the formula

$$
\phi_{i j}^{A}=\oint \rho_{x} \pi_{i}^{A} \pi_{j A} \cdot \hat{n}^{A} f(y, z) \Delta \pi,
$$

with $\hat{\eta}^{A}=-\partial / \partial \eta_{A}$. This is likewise an $\mathrm{SU}(2)$ singlet state.

\footnotetext{
*In the case of zero rest mass 2-twistor particles, it is the magnitude of the helicity that coincides with the leptonic isospin.
} 
Note that in the analysis above we have regarded the left and right hand parts of the electron as being in some sense very distinct fields. This is apparently the view that must be taken if one is to regard the mass of the electron as arising by way of the Higgs mechanism. In the approximation where we ignore the coupling of the electron to the Higgs scalars, the electron wave function splits into a pair of independent massless spin 1/2 fields, one of each helicity. This view is consonant with certain aspects of the general outlook on physics suggested by complex analytic geometry, according to which one regards massless fields as being more basic, with mass arising in a secondary way as a consequence of interactions between the various fields. As for the Higgs bosons, the relevant multiplet structure is given as follows:

$$
\phi_{j k}^{i}(x)=\oint \rho_{x} \pi_{j A} \cdot \hat{\pi}_{k}^{A} \hat{\pi}^{A i} \hat{\eta}_{A} f(y, z) \Delta \pi,
$$

which is an isodoublet since it is skew over the indices $\mathrm{j}$ and $\mathrm{k}$. The corresponding antiparticle doublet is

$$
\phi_{i}^{j k}(x)=\oint \rho_{x} \hat{\pi}^{j A} \hat{\pi}_{A}^{k} \pi_{A} i^{n} n^{A^{\prime}} f(y, z) \Delta \pi
$$

And, finally, the quartet of intermediate vector bosons is given by the spin-one part of the expression:

$$
\phi_{i}^{j A A^{\prime}}(x)=\oint \rho_{x} \hat{\pi}^{j A} \pi_{i}^{A} A^{\prime} f(y, z) \Delta \pi,
$$

the mixing angle $\theta_{\mathrm{w}}$ between $\mathrm{w}^{\circ}$ and $\mathrm{z}^{\circ}$ being left free for the moment.

It is not difficult to construct appropriate expressions for the relevant quantum operators for determining the quantum numbers of the states described above. One can write down the holomorphic differential operators corresponding to mass, spin, electric charge, leptonic hypercharge, lepton number, and leptonic isospin, thereby arriving at a set of operators sufficient for the description of states arising in the Weinberg-Salam model; these will be presented in detail elsewhere.

Now we come to the problem of incorporating muons and other leptons into the picture. One standard approach to the problem of muons is the take a 'xerox copy' of the whole of the set-up above (for the fermions) and assign it a new flavour quantum number 
called muon number. What remains unsatisfactory about this procedure is that it is so arbitrary. The 3-twistor Weinberg-Salam scheme, however, is suggestive of a new approach to the xerox copy problem: the point is that although we have at our disposal. now (as described above) a set of operators sufficient for the Weinberg-Salam theory, we have not yet exhausted the internal degrees of freedom of the 3-twistor system in terms of which the leptons are being represented.

In fact, there remain yet two further internal degrees of freedom for a 3-twistor system, corresponding to the two SU(3) Casimir operators for the three twistors $\mathrm{Y}^{\alpha}$ and $\mathrm{z}_{i}^{\alpha}$. By exploiting these additional degrees of freedom it is possible to distinguish between distinct 3 -twistor states which otherwise have identical Weinberg-Salam multiplet structures. To put it another way, if one specifies the Weinberg-Salam quantum numbers (mass, spin, charge, leptonic hypercharge, lepton number, and leptonic isospin), these alone do not suffice to uniquely characterize the contour integral formulae shown in equations 11-17. One must in addition specify the eigenvalues of the SU(3) Casimir operators. In equations 11-17 the SU(3) eigenvalues are in fact the 'simplest' compatible with the states indicated. However, by choosing higher SU(3) representations one can generate new states within the 3twistor framework which are 'xerox copies' of the original Weinberg-Salam multiplets.

SU(3) representations are most conveniently characterized by a pair of non-negative integers $\{\lambda, \mu\}$. After a judicious application of $\mathrm{SU}(3)$ epsilons, any irreducible $\mathrm{SU}(3)$ tensor can be brought into a form where it is tracefree and totally symmetric over both its upstairs and downstairs indices; then $\lambda$ is the number of upstairs indices and $\mu$ is the number of downstairs indices. According to this scheme the $e_{L}^{-}$isodoublet belongs to a $\{1,0\}$ representation of $\mathrm{SU}(3)$, and the $e_{\mathrm{R}}^{-}$isosinglet belongs to a $\{0,2\}$ representation. Similarly, the $e_{R}^{+}$isodoublet belongs to a $\{0,1\}$ and the $e_{L}^{+}$isosinglet belongs to a $\{2,0\}$.

For the muonic leptons it is natural to suppose that the $\mu_{L}^{-}$ isodoublet belongs to a $\{2,1\}$ representation of $\mathrm{SU}(3)$, and that the $\mu_{R}^{-}$isosinglet belongs to a $\{1,3\}$. The corresponding CP-con- 
jugate states belong to repesentations given, as before, by interchanging $\lambda$ and $\mu$.

With these examples in mind it is not difficult to discern the general pattern. We have a sequence of lepton "generations" $\Lambda(n)$, such that for any given value of $n$ the $\Lambda_{L}^{-}$isodoublet belongs to an $\{n, n-1\}$, the $\Lambda_{R}^{+}$isodoublet belongs to an $\{n-1, n\}$, the $\Lambda_{L}^{+}$ isosinglet belongs to an $\{n+1, n-1\}$, and the $\Lambda_{R}^{-}$isosinglet belongs to an $\{n-1, n+1\}$. It is not unreasonable to hypothesize that the $\tau$-particle and its family comprise the $n=3$ generation; it should be clear, however, that some modification of the scheme would be called for if it emerges (as it very well might) that any of the neutrinos are not zero rest mass particles.

The picture outlined above is only one of several possible approaches one might adopt towards leptons within the context of twistor theory; I believe that of all the approaches put forward thus far it agrees most readily with the 'standard model' of Weinberg and Salam. Other possibilities, however, are certainly not out of the question, and one should not be left with the impression that the 3-twistor scheme is in any sense the last word on the subject. It does, nevertheless, indicate something of the scope for model building available within twistor theory, and illustrates the curious interplay between the twistor internal symmetry groups and their phenomenological counterparts.

\section{HADRONS}

As with the case of leptons, more than one approach within the framework of twistor theory can be developed towards the problem of the structure and classification of hadrons. The most elementary approach is to regard hadron states as holomorphic functions of three twistors - mathematically the scheme is quite similar to the one described earlier for leptons, but the assignment of observables is different. Here to simplify matters we are temporarily ignoring charmed particles- the U(3) group associated with the three twistors refers to the three most primitive flavour degrees of freedom (i.e. baryon number, electric charge, and hypercharge). 
Let us label the three twistors $z_{i}^{\alpha}(i=1,2,3)$ according to the scheme:

$$
z_{i}^{\alpha}=\left(u^{\alpha}, D^{\alpha}, s^{\alpha}\right),
$$

and for the three operators $\hat{\mathrm{z}}_{\alpha}^{i}$ write:

$$
\hat{\mathrm{z}}_{\alpha}^{i}=\left(\hat{\mathrm{U}}_{\alpha}, \hat{\mathrm{D}}_{\alpha}, \hat{\mathrm{s}}_{\alpha}\right)
$$

Then for the baryon number $\hat{\mathrm{B}}$, the electric charge $\hat{\mathrm{Q}}$, and the hypercharge $\hat{\mathrm{Y}}$ we have the following operator expressions:

$$
\begin{aligned}
& \hat{B}=-\frac{1}{3}\left(U^{\alpha} \hat{U}_{\alpha}+D^{\alpha} \hat{D}_{\alpha}+s^{\alpha} \hat{\mathrm{S}}_{\alpha}\right)+2 \\
& \hat{\mathrm{Q}}=-\frac{1}{3}\left(2 \mathrm{U}^{\alpha} \hat{\mathrm{U}}_{\alpha}-D^{\alpha} \hat{\mathrm{D}}_{\alpha}-\mathrm{s}^{\alpha} \hat{\mathrm{S}}_{\alpha}\right) \\
& \hat{\mathrm{Y}}=-\frac{1}{3}\left(\mathrm{U}^{\alpha} \hat{\mathrm{U}}_{\alpha}+D^{\alpha} \hat{\mathrm{D}}_{\alpha}-2 \mathrm{~s}^{\alpha} \hat{\mathrm{S}}_{\alpha}\right)
\end{aligned}
$$

The operator for total isospin is:

$$
\hat{I}^{2}=\left(\hat{I}_{1}\right)^{2}+\left(\hat{I}_{2}\right)^{2}+\left(\hat{I}_{3}\right)^{2} \text {, }
$$

where

$$
\begin{aligned}
& \hat{I}_{1}=-\frac{1}{2}\left(U^{\alpha} \hat{D}_{\alpha}+D^{\alpha} \hat{U}_{\alpha}\right) \\
& \hat{I}_{2}=-\frac{i}{2}\left(U^{\alpha} \hat{D}_{\alpha}-D^{\alpha} \hat{U}_{\alpha}\right) \\
& \hat{I}_{3}=-\frac{1}{2}\left(U^{\alpha} \hat{U}_{\alpha}-D^{\alpha} \hat{D}_{\alpha}\right) .
\end{aligned}
$$

These operators satisfy the usual commutation relations for SU(2), and it is straightforward to verify the familiar identity

$$
\hat{Q}=\hat{I}_{3}+\hat{Y} / 2 \text {. }
$$

The mass-squared operator is

$$
M^{2}=I_{\alpha \beta} I^{\gamma \delta} z_{i}^{\alpha}{ }_{j}^{\beta} \hat{z}_{\gamma}^{i} \hat{z}_{\delta}^{j}
$$

and the operator for spin is a similar expression of a somewhat more complicated nature (cf. ref. 11, p. 193). The two SU(3) Casimir operators are

$$
\begin{aligned}
& \hat{C}_{2}=A_{i}^{[i} A_{j}^{j]} \\
& \left.\hat{C}_{3}=A_{i}^{[i} A_{j}^{j} A_{k}^{k}\right]
\end{aligned}
$$


with

$$
A_{j}^{i}=z_{j}^{\alpha} \hat{z}_{\alpha}^{i}-\frac{1}{3} \delta_{j}^{i} z_{k}^{\alpha} \hat{z}_{\alpha}^{k}
$$

As with the lepton scheme discussed earlier, SU(3) representations can be conveniently characterized by a pair of integers $\{\lambda, \mu\}$. These are related to the eigenvalues of $\hat{C}_{2}$ and $\hat{C}_{3}$ by the formulae

$$
\begin{aligned}
& c_{2}=\frac{1}{3}\left(\lambda^{2}+\mu^{2}+\lambda \mu+3 \lambda+3 \mu\right) \\
& c_{3}=\frac{1}{27}(\lambda-\mu)(2 \lambda+\mu+3)(\lambda+2 \mu+3)
\end{aligned}
$$

Three-twistor states are constrained by a certain set of inequalities, derived by Perjés and Sparling ${ }^{11}$. These are:

$$
\begin{aligned}
& \frac{2 \lambda+\mu}{3} \geq J+\frac{B}{2} \geq \frac{\mu-\lambda}{3} \\
& \frac{\lambda+2 \mu}{3} \geq J-\frac{B}{2} \geq \frac{\lambda-\mu}{3} .
\end{aligned}
$$

A short calculation establishes that these relations imply:

$$
\mathrm{J} \leq \frac{\lambda+\mu}{2},
$$

and also

$$
\frac{\lambda+2 \mu}{3} \geq \mathrm{B} \geq \frac{-2 \lambda-\mu}{3} \text {. }
$$

The relation (29) puts a bound on the spin for any fixed representation of $\mathrm{SU}(3)$. It is on account of this relation that it is not possible to develop a satisfactory theory of hadron resonances within a 3-twistor context - it is necessary, as will be discussed below, to seek out representations of a more elaborate character.

The inequalities in formula (30) restrict the values B can take on within a given $\mathrm{SU}(3)$ representation. One can check that (28), (29) and (30) are in fact compatible with the existence of a 3-twistor description of a $J=\frac{1}{2}$ octet and a $J=3 / 2$ decimet, each with $B=1$. Taken together these two multiplets comprise the basic baryon 56-supermultiplet, for which the relevant contour integral formula is

$$
\phi_{A B C}^{i j k}(x)=\oint \rho_{x} \hat{\pi}_{A}^{i} \hat{\pi}_{B}^{j} \hat{\pi}_{C}^{k} f(z) \Delta \pi \text {. }
$$


An interesting and perhaps not fully understood feature of this description is that the correct group and spin symmetries for a 56-plet emerge automatically within the 3-twistor framework, without one having to introduce colour degrees of freedom.

As indicated above, the characterization of higher hadron resonances requires us to introduce models of a more intricate nature. One approach is to employ functions of six twistors*, regarding baryon resonances as bound states of a quark and a diquark - three of the twistors correspond to the quark, and three to the diquark. The quarks and diquarks in this scheme are colourless, and the diquarks are single particle states - not bound states of pairs of quarks. The model has been explored in some depth, and is described in references 10, 13, 15 and 16.

\section{A 6-TWISTOR GEORGI-GLASHOW SCHEME}

Another line of development relevant to the description of hadrons involves the incorporation of grand unified theories into a twistor framework. This approach has been rather successful, and I shall outline below a scheme devised recently by T.R. Hurd and myself.

The simplest and at present perhaps most convincing example of a grand unified theory is the celebrated su(5) model of Georgi and Glashow ${ }^{22}$. It contains $\mathrm{n}$ fundamental fermion families, $\mathrm{n}$ being 3 or more. Each family consists of: 2 flavours of quarks, each in 3 colours and 2 helicities; 1 lepton in 2 helicites, together with a matching neutrino in 1 helicity- plus the antiparticles of all the above. Each family has 30 states, consisting of a $\underline{5}, \mathrm{a} \underline{5}^{*}, \mathrm{a} \underline{10}$, and a $\underline{10}^{*}$. The electron family, for example, is given the following multiplet structure (ignoring Cabibbo effects):

\footnotetext{
*The original motivation for the 6-twistor scheme came from closely related work done by G.A.J. Sparling in 1975.
} 


$$
\left(\begin{array}{c}
d_{B} \\
d_{R} \\
d_{G} \\
e^{+} \\
\bar{v}_{e}
\end{array}\right)_{\text {Right. }}\left(\begin{array}{ccccc}
0 & \bar{u}_{G} & \bar{u}_{R} & u_{B} & d_{B} \\
\cdot & 0 & \bar{u}_{B} & u_{R} & d_{R} \\
\cdot & \cdot & 0 & u_{G} & d_{G} \\
\cdot & \cdot & \cdot & 0 & e^{+}
\end{array}\right)_{\text {Left }}
$$

together with the corresponding CP-conjugate states; the 5-by-5 matrix is skew.

The particle content of the Georgi-Glashow model can be represented rather naturally using functions of six twistors, this representation being in fact a straightforward generalization of the Weinberg-Salam picture presented in section 5 . If the six twistors are labelled $\left(\mathrm{y}^{\alpha}, \mathrm{z}_{i}^{\alpha}\right)$ with $i=1 \ldots 5$, then the righthanded quintet is determined by the formula:

$$
\phi_{i}^{A^{\prime}}=\oint \rho_{x} \pi_{i}^{A^{\prime}} f(y, z) \Delta \pi,
$$

and the left-handed decimet is given by:

$$
\phi_{i j}^{A}=\oint \rho_{x} \pi_{i}^{A} \pi_{j A} \cdot \hat{n}^{A} f(y, z) \Delta \pi,
$$

the matching $\mathrm{CP}$-conjugate states being straightforward to construct. The SU(5) 24-plet given by the tracefree part of

$$
\phi_{i}^{j A A^{\prime}}=\oint \rho_{x} \hat{\pi}^{j A} \pi_{i}^{A^{\prime}} f(y, z) \Delta \pi
$$

splits into a $\underline{24}$ of intermediate vector bosons, and a $\underline{24}$ of scalar Higgs bosons. The model also requires a second set of Higgs particles, given by:

$$
\phi_{i}^{j k}=\oint \rho_{x} \hat{\pi}^{j A} \hat{\pi}_{A^{k} \pi^{\prime} i^{\prime}} n^{A^{\prime}} f(y, z) \Delta \pi,
$$

which splits naturally into a $\underline{5}$ and a $\underline{45}$.

The multiplets indicated above include the "first generation" of fermions, i.e. the electron-type leptons, together with the up and down type quark states. As with the case of leptons the scheme contains further degrees of freedom, and it a possible to incorporate additional generations of fermions (with no apparent bound on the number of such generations) by means of a similar method. Thus we see that the 'standard' theories of 
strong, weak and electromagnetic interactions can be fitted rather neatly - at least in a provisional way - into a twistorial framework.

\section{Acknowledgements}

I would like to express my gratitude to colleagues at the Mathematical Institute, Oxford, particularly T.R. Hurd, A.S. Popovich and R. Penrose, for useful discussion in connection with the material presented here. The final draft of the manuscript was prepared during the author's visit to the Central Research Institute for Physics, Budapest, in March 1980; I am very grateful to $\mathrm{Z}$. Perjés and other colleagues there for their hospitality. This work has been supported by a grant from the U.K. Science Research Council, and a Junior Research Fellowship at Wolfson College, Oxford.

\section{REFERENCES}

1. R. Penrose and M.A.H. MacCallum, Phys. Reports, 6C 241 (1972).

2. Z. Perjés, Phys. Rev., 11D, 2031 (1975).

3. R. Penrose, "Twistor Theory, its Aims and Achievements" in Quantum Gravity, edited by C.J. Isham, R. Penrose, and D.W. Sciama (Oxford University Press, 1975).

4. R. Penrose, "Twistors and Particles", in Quantum Theory and the Structure of Time and Space, edited by L. Castell, M. Drieschner and C.F. von Weizsäcker (Carl Hanser Verlag, 1975).

5. R. Penrose, Reports Math. Phys., 12, 65 (1977).

6. A.P. Hodges, "The Description of Mass in the Theory of Twistors", Ph.D. Thesis, Birkbeck College, London (1975).

7. K.P. Tod, "Massive Particles with Spin in General Relativity and Twistor Theory", D. Phil. Thesis, University of Oxford (1975).

8. K.P. Tod, Reports Math. Phys. 11, 339 (1977).

9. Z. Perjés, Reports Math. Phys. 12, 193 (1977). 
10. A.S. Popovich, "Twistor Classification of Elementary Particles", M.Sc. Thesis, University of Oxford (1978).

11. Z. Perjés and G.A.J. Sparling, $\$ 4.2$ in Advances in Twistor Theory, edited by L.P. Hughston and R.S. Ward (Pitman, 1979).

12. R. Penrose and G.A.J. Sparling, $\$ 4.7$ in Advances in Twistor Theory, edited by L.P. Hughston and R.S. Ward (Pitman, 1979).

13. M.C. Sheppard, "Aspects of Twistor Particle Theory, D.Phil. Thesis, University of Oxford (1979).

14. L.P. Hughston, "A Particle Classification Scheme Based on the Theory of Twistors", D. Phil. Thesis, University of Oxford $(1976)$.

15. L.P. Hughston, Twistors and Particles, Lecture Notes in Physics, Volume 97 (Springer-Verlag, 1979).

16. L.P. Hughston, $\S 4.1$ \& 4.8 in Advances in Twistor Theory, edited by L.P. Hughston and R.S. Ward (Pitman, 1979).

17. Z. Perjés, Phys. Rev. D 20, 1857 (1979).

18. R. Penrose, Int. J. Theor. Phys., Vol. 1, No. 1, 61 (1968).

19. S. Weinberg, Phys. Rev. Letts. 19, 1264 (1967).

20. S. Weinberg, Phys. Rev. Letts. 28, 1688 (1971).

21. A. Salam, in Elementary Particle Physics, edited by N.Svartholm (Almguist and Wilksells, Stockholm, 1968).

22. H. Georgi and S. Glashow, Phys. Rev. Letts. 32, 438 (1974). 


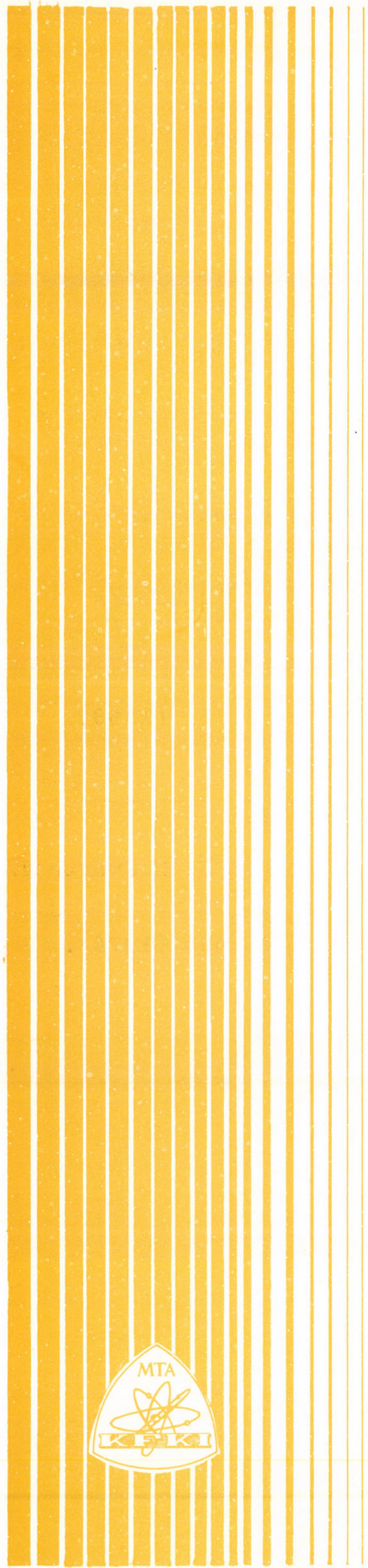

Kiadja a Központi Fizikai Kutató Intézet Felelōs kiadó: Szegõ Károly

Szakmai lektor: Perjés zoltán

Nyelvi lektor: Lovas István

Példányszám: 225 Törzsszám: 80-212

Készült a KFKI sokszorositó ủzemében Budapest, 1980. április hó 